\title{
NEW SUFFICIENT CONDITIONS FOR THE APPROXIMATION OF DISTINCT SOLUTIONS OF THE QUADRATIC EQUATION IN BANACH SPACES
}

\author{
IOANNIS K. ARGYROS
}

\begin{abstract}
Using the "theory of majorants" we provide new sufficient conditions for the approximation of distinct solutions of the quadratic equation in Banach spaces. Our results are applied to a Riccati ordinary differential equation.
\end{abstract}

1. Introduction.

Consider the equation

$$
x=y+B(x, x)
$$

in a Banach space $E$ over the filed $\mathbb{R}$ of real numbers, where $B: E \times E \rightarrow \widehat{E}$ is a bounded bilinear operator with values in a Banach space $\widehat{E}$ and $y \in E$ is fixed. We introduce the iteration

$$
x_{n+1}=B\left(x_{n}\right)^{-1}\left(x_{n}-y\right), \quad n=0,1,2, \cdots
$$

for approximating solutions $x^{*}$ of equation (1). For each fixed $x \in E, B(x)$ denotes a linear operator from $E$ to $\widehat{E}$ such that $B(x)(y)=B(x, y)$, for all $y \in E$.

Received October 8, 1990.

A. M. S. (1980) Classification codes: 47D15, 47H17, 65R20, 65J10.

Key words and phrases: Banach space, quadratic equation. 
Special cases of (1) appear in many interesting problems arising in astrophysics, in the kinetic theory of gases as well as the theory of ordinary and partial differential equations [4], [5]. Equation (1) has been studied extensively. The continued fraction approach [3], [8], the contraction mapping theorem technique [1], [2], [9], [10] and the famous Newton-Kantorovich method [6], [7], [9] have been used to find a solution $x^{*}$ of equation (1).

A common hypothesis for the above techniques is the estimate $4\|B\|\|y\|<1$.

It turns out that under this hypothesis the previous mentioned techniques approximate a small solution $v^{*}$ of equation (1) for any starting point $x_{0}$ close enough to the solution. The obtained solution $v^{*}$ is such that $v^{*}=v^{*}(y) \rightarrow 0$ as $y \rightarrow 0$. We make use of the "theory of majorants" [6], [7] and under assumptions similar to the ones introducedd in the above mentioned techniques, iteration (2) can be used to approximate a second solution $x^{*}$ of (1) with $x^{*} \neq v^{*}$ and $x^{*}=x^{*}(y) \rightarrow 0$ as $y \rightarrow 0$. Moreover, under the same assumptions we show that the Newton-Kantorovich method [7] can bo used io obtain a solution $z_{N}^{*}=x^{*}$ also. This result is not known not even for quadratic systems in $\mathbb{R}^{n}, n>1$. Some sufficient conditions are also given for the existence of more than one distinct solutions of (1). Our results are illustrated with the solution of a quadratic system in $E=\mathbb{R}^{2}$ as well as the solution of a Riccati differential equation.

\section{The "MAJORANT THEORY" and Equation (1)}

Definition 1. An operator $B: E_{1} \times E_{2} \rightarrow E_{3}$ is called bilinear if it is linear in each variable separately and symmetric if $E_{1}=E_{2}$ and $B(x, y)=B(y, x)$ for all $(x, y) \in E_{1} \times E_{2}$.

Definition 2. The mean $\bar{B}$ of $B$ on $E_{1} \times E_{2}$ is defined by

$$
\bar{B}(x, y)=\frac{1}{2}(B(x, y)+B(y, x)) \text { for all }(x, y) \in E_{1} \times E_{2}
$$

Definition 3. A bilinear operator $B: E_{1} \times E_{2} \rightarrow E_{3}$ is said to be bounded 
if there exists $q>0$ such that

$$
\|B(x, y)\| \leq q\|x\| \cdot\|y\| \text { for all }(x, y) \in E_{1} \times E_{2} \text {. }
$$

The quantity $\|B\|=\sup _{\|x\| \leq 1,\|y\| \leq 1}\|B(x, y)\|$ is called the norm of $B$. Note that, for $B$ symmetric,

$$
\vec{B}(x, x)=B(x, x) \text { for all } x \in E_{1} \text {. }
$$

Without loss of generality due to (3) we may assume that the operator $B$ in (1) is symmetric.

From now on $E_{1}=E_{2}=E$ and $E_{3}=\widehat{E}$. We can now prove a theorem for the existence of a solution $x^{*}$ of equation (1).

Theorem 1. Let $B$ be a bounded symmetric bilinear operator on $E \times E$ and suppose that $x_{0}, y \in E$ with $x_{0} \neq 0$ and $x_{0} \neq y$. Assume:

(i) The inverse of the linear operator $B\left(x_{0}\right): E \rightarrow E$ with $B\left(x_{0}\right)(x)=B\left(x_{0}, x\right)$ for all $x \in E$ exists and is bounded.

(ii) The estimates:

$$
0 \leq c<1
$$

and

$$
0 \leq d<\frac{(1-c)^{2}}{4 a b}
$$

are true, where we have denoted

$$
\begin{gathered}
a \geq\left\|B\left(x_{0}\right)^{-1}\right\|, \\
b \geq\|B\|, \\
c \geq\left\|B\left(x_{0}\right)^{-1}\left(I-B\left(x_{0}\right)\right)\right\|
\end{gathered}
$$

and

$$
d \geq\left\|B\left(x_{0}\right)^{-1}\left(B\left(x_{0}, x_{0}\right)+y-x_{0}\right)\right\|
$$


Then: (a) The real sequence $\left\{t_{n}\right\}, n=0,1,2, \cdots$ given by

$$
\begin{gathered}
t_{n+2}=t_{n+1}-\frac{1+c-2 a b t_{n+1}}{1+c+2 a b t_{n+1}}\left(t_{n}-t_{n+1}\right), \quad n=0,1,2, \cdots, \\
t_{0}=\frac{1-c}{2 a b}, \quad t_{1}=\left[\frac{1+c}{2}\right] t_{0},
\end{gathered}
$$

is positive and decreasingly converges to zero.

(b) The sequence $\left\{x_{n}\right\}, n=0,1,2, \cdots$ generated by (2) is well defined, remains in $U\left(x_{0}, r_{0}\right)=\left\{x \in E /\left\|x-x_{0}\right\|<r_{0}=\frac{1-c}{2 a b}\right\}$ and converges to a unique solution $x^{*} \in \bar{U}\left(x_{0}, r_{0}\right)$ of equation (1).

Moreover, the following estimates are true for all $n=0,1,2, \cdots$,

$$
\left\|x_{n+1}-x_{n}\right\| \leq t_{n}-t_{n+1}
$$

and

$$
\left\|x_{n}-x^{*}\right\| \leq t_{n} \leq\left[\frac{1+c}{2}\right]^{n} t_{0}
$$

Proof. (a) It can easily be seen by (10) that the sequence $\left\{t_{n}\right\}, n=$ $0,1,2, \cdots$ is certainly nonnegative if

$$
(1+c) t_{k+1}+2 a b t_{k} t_{k+1}-(1+c) t_{k} \geq 0 \text { for all } k=0,1,2, \cdots
$$

Inequality (11) is true as equality for $k=0$. Let us assume that it is true for $k=0,1,2, \cdots, n$. We shall show that it is ture for $k=n+1$. Using (10), the left hand side of inequality (11) for $k=n+1$ becomes

$$
\frac{2 a b\left(1+c+2 a b t_{k}\right) t_{k+1}^{2}+(1+c)^{2} t_{k+1}-(1+c)^{2} t_{k}}{1+c+2 a b t_{k+1}}
$$

which is nonnegative if $t_{k+1} \geq \frac{(1+c) t_{k}}{1+c+2 a b t_{k}}$ and that is true by our assumption.

By the choice of $t_{0}, t_{1}$ and $(9), t_{0}-t_{1}>0$.

Let us assume that

$$
t_{k}-t_{k+1}>0, \quad k=0,1,2, \cdots, n \text {. }
$$


Using (10), we see that (12) is true for $k=n+1$ if

$$
1+c-2 a b t_{k+1}>0 \text { for } k=0,1,2, \cdots, n \text {. }
$$

Inequality (13) is true for $k=0$ by the choice of $t_{1}$. Let us assume that (13) is ture for $k=0,1,2, \cdots, n$. To show (13) for $k=n+1$ it suffices to show $t_{k+2}<\frac{1+c}{2 a b}$ or by $(10)$

$$
2 a b\left[2(1+c) t_{k+1}+2 a b t_{k} t_{k+1}-(1+c) t_{k}\right] \leq(1+c)^{2}+2 a b(1+c) t_{k+1}
$$

or $t_{k+1} \leq \frac{1+c}{2 a b}$ which is true by hypothesis.

We have now showed that the real sequence $\left\{t_{n}\right\}, n=0,1,2, \cdots$ is positive and decreasing and as such it converges to some $t^{*} \geq 0$. But using simple induction and (10) we can easily show that $t_{n+1} \leq\left[\frac{1+c}{2}\right] t_{n} \leq\left[\frac{1+c}{2}\right]^{n+1} t_{0}$. That is $t^{*}=0$.

(b) Let us observe that the linear operator $B(x)$ is invertible for all $x \in$ $U\left(x_{0}, r_{0}\right)$. Indeed we have

$$
\left\|B\left(x_{0}\right)^{-1} B\left(x-x_{0}\right)\right\| \leq\left\|B\left(x_{0}\right)^{-1}\right\| \cdot\|B\| \cdot\left\|x-x_{0}\right\| \leq a b\left\|x-x_{0}\right\|<1
$$

so that according to Banach's lemma on invertible operators

$$
\left\|B(x)^{-1}\right\|=\left\|\left[I+B\left(x_{0}\right)^{-1} B\left(x-x_{0}\right)\right]^{-1} B\left(x_{0}\right)^{-1}\right\| \leq \frac{a}{1-a b\left\|x-x_{0}\right\|} .
$$

We shall prove that

$$
\left\|x_{n}-x_{n+1}\right\| \leq t_{n}-t_{n+1} \quad \text { for } \quad n=0,1,2, \cdots .
$$

By (a) it follows that if (2) is well defined for $n=0,1,2, \cdots, k$ and if (15) holds for $n \geq k$ then $\left\|x_{0}-x_{n}\right\| \leq t_{0}-t_{n}<t_{0}-t^{*}$ for $n \leq k$. This shows that (14) is satisfied for $x=x_{i}, i \leq k$. Thus (2) will be defined for $n=k+1$, too. By (2) and (9) $\left\|x_{1}-x_{0}\right\| \leq d \leq t_{0}-t_{1}$. That is, (15) is true for $n=0$. Suppose (15) 
holds for $n=0,1,2, \cdots, k$. Observing that

$$
\begin{aligned}
& B\left(x_{k+1}\right)\left(x_{k+1}-x_{k+2}\right) \\
= & B\left(x_{k+1}, x_{k+1}\right)+y-x_{k+1}-B\left(x_{k}, x_{k}\right)-y+x_{k}+B\left(x_{k}\right)\left(x_{k}-x_{k+1}\right) \\
= & B\left(x_{k+1}-x_{k}, x_{k+1}+x_{k}\right)+x_{k}-x_{k+1}+B\left(x_{k}\right)\left(x_{k}-x_{k+1}\right) \\
= & B\left(x_{k+1}-x_{k}, x_{k+1}-x_{k}\right)+B\left(x_{k}-x_{k+1}, x_{k+1}-x_{k}\right)+B\left(x_{k+1}, x_{k+1}-x_{k}\right) \\
& \quad-\left(x_{k+1}-x_{k}\right) \\
= & \left(B\left(x_{k+1}\right)-I\right)\left(x_{k+1}-x_{k}\right),
\end{aligned}
$$

we get

$$
x_{k+1}-x_{k+2}=B\left(x_{k+1}\right)^{-1}\left[B\left(x_{k+1}-x_{0}\right)+B\left(x_{0}\right)-I\right]\left(x_{k+1}-x_{k}\right) .
$$

By taking norms in (16) and using (14) we obtain

$$
\left\|x_{k+1}-x_{k+2}\right\| \leq \frac{\left[c+a b\left(t_{0}-t_{k+1}\right)\right]\left(t_{k}-t_{k+1}\right)}{1-a b\left(t_{0}-t_{k+1}\right)}=t_{k+1}-t_{k+2}
$$

by choice of $t_{0}$. Inequality (15) shows that $\left\{x_{n}\right\}, n=0,1,2, \cdots$ is a Cauchy sequence in a Banach space $E$ and as such it converges to some $x^{*} \in E$. By taking the limit as $n \rightarrow \infty$ in (2) we get $x^{*}=y+B\left(x^{*}, x^{*}\right)$. That is $x^{*}$ is a solution of equation (1). Fix $n$ and let $p=0,1,2, \cdots$. Then

$$
\left\|x_{n}-x^{*}\right\| \leq\left\|x_{n}-x_{n+p}\right\|+\left\|x_{n+p}-x^{*}\right\| \leq t_{n}-t_{n+p}+\left\|x_{n+p}-x^{*}\right\| .
$$

By letting $p \rightarrow \infty$ we obtain

$$
\left\|x_{n}-x^{*}\right\| \leq t_{n}-t^{*}, \quad n=0,1,2, \cdots .
$$

By (18) for $n=0$ we get

$$
\left\|x_{0}-x^{*}\right\| \leq t_{0}-t^{*}=\frac{1-c}{2 a b}-t^{*}=\frac{1-c}{2 a b} .
$$

That is $x^{*} \in \bar{U}\left(x_{0}, r_{0}\right)$.

Finally, let us assume that there exists a second solution $z^{*} \in U\left(x_{0}, r_{0}\right)$ of equation (1). 
By (2) we have

$$
\begin{aligned}
x_{n+1}-z^{*} & =x_{n}-B\left(x_{n}\right)^{-1}\left(y+B\left(x_{n}, x_{n}\right)-x_{n}\right)-z^{*} \\
& =B\left(x_{n}\right)^{-1}\left[B\left(x_{n}\right)\left(x_{n}-z^{*}\right)+x_{n}-y-B\left(x_{n}, x_{n}\right)+y+B\left(z^{*}, z^{*}\right)-z^{*}\right] \\
& =-B\left(x_{n}\right)^{-1}\left[B\left(z^{*}-z_{0}\right)+B\left(x_{0}\right)-I\right]\left(x_{n}-z^{*}\right) .
\end{aligned}
$$

By taking the norms in the above identity and using (14) we obtain

$$
\left\|x_{n+1}-z^{*}\right\| \leq 2\left[\frac{c+a b\left\|z^{*}-x_{0}\right\|}{1+c+2 a b t_{n+1}}\right]\left\|x_{n}-z^{*}\right\|
$$

By the choice of $r_{0}$ the factor of $\left\|x_{n}-z^{*}\right\|$ is less than 1 so that $\left\|x_{n}-z^{*}\right\|$ goes to zero as $n \rightarrow \infty$; hence $z^{*}=\lim _{n \rightarrow \infty} x_{n}=x^{*}$.

That completes the proof of the theorem.

Moreover, we can show the following theorem:

Theorem 2. Let $B$ be a bounded symmetric bilinear operator on $E \times E$ and suppose that $x_{0}, y \in E$ with $x_{0} \neq 0, x_{0} \neq y$. Assume:

(i) The following estimate is true

$$
4 b e<1
$$

where

$$
e \geq\|y\|
$$

(ii) The hypotheses of Theorem 1 are satisfied for some $x_{0} \in \mathbb{E}$ such that

$$
\left\|x_{0}\right\|>p \quad \text { with a certain } p \in\left(p_{1}, p_{2}\right)
$$

where $p_{1}$ and $p_{2}$ are the two positive solutions of the scalar quadratic equation

$$
b z^{2}-z+e=0
$$

Then:

(a) The iteration

$$
v_{n+1}=y+B\left(v_{n}, v_{n}\right)
$$


remains in $U\left(0, p_{1}\right)$ and converges to a unique solution $v^{*}$ of equation (1) in $U\left(0, \frac{1}{2\|B\|}\right)$ for any $v_{0} \in \bar{U}\left(0, p_{1}\right)$. Moreover, for all $n=0,1,2, \cdots$

$$
\left\|v_{n}-v^{*}\right\| \leq p_{1}-e \sum_{j=0}^{n} \frac{(2 j) !}{j !(j+1) !}(e b)^{j} .
$$

(b) The solution $x^{*}$ of equation (1) obtained via iteration (2) is such that $x^{*} \neq v^{*}$.

Proof. (a) The first part of the result in (a) follows from Corollary 1 in [1], whereas the second part follows from Theorem 18 in [10].

(b) We shall show that $\left\|x_{n}\right\|>p$ for a certain $p \in\left(p_{1}, p_{2}\right)$. By (2) we obtain $\left\|x_{n}-y\right\|=\left\|B\left(x_{n}, x_{n+1}\right)\right\| \leq\|B\| \cdot\left\|x_{n}\right\|\left\|x_{n+1}\right\|$ or

$$
\left\|x_{n+1}\right\| \geq \frac{\left\|x_{n}-y\right\|}{\|B\| \cdot\left\|x_{n}\right\|}
$$

Assume that $\left\|x_{k}\right\|>p$ for all $k=0,1,2, \cdots, n$. Since

$$
\left\|x_{n}\right\|>p>e
$$

it is enough to show

$$
\frac{\left\|x_{n}\right\|-e}{b\left\|x_{n}\right\|}>p
$$

or

$$
\left\|x_{n}\right\|>\frac{e}{1-p b} .
$$

By (24) it finally suffices to show $p>\frac{e}{1-p b}$ which is true for $p \in\left(p_{1}, p_{2}\right)$. By taking the limit as $n \rightarrow \infty$ in (24) we get $\left\|x^{*}\right\| \geq p$. Therefore, we obtain $x^{*} \neq v^{*}$.

That completes the proof of the theorem.

Furthermore, we can prove the following theorem concerning the number of solutions of equation (1).

Theorem 3. Let $B$ be a bounded symmetric bilinear operator on $E \times E$ and suppose that $x_{0}, y \in E$ with $x_{0} \neq y$ and $y \neq 0$. 
Assume:

(i) the point $x_{0} \in \mathbb{E}$ is such that

$$
B\left(x_{0}\right)=I
$$

(ii) the inequality (19) is true.

Then the elements $v^{*}, x^{*}, x_{0}-x^{*}$ and $x_{0}-v^{*}$ are solutions of equation (1) with

$$
x^{*} \neq x_{0}-x^{*}
$$

and

$$
v^{*} \neq x_{0}-v^{*}
$$

Proof. It follows by (i) that the hypotheses of Theorem 1 are satisfied. That is $x^{*}$ is a solution of equation (1). By (ii) $v^{*}$ is a solution of equation (1). For $z=x_{0}-x^{*}$ we have

$$
\begin{aligned}
y+B(z, z) & =y+B\left(x_{0}-x^{*}, x_{0}-x^{*}\right)=y+B\left(x_{0}, x_{0}\right)-2 B\left(x_{0}, x^{*}\right)+B\left(x^{*}, x^{*}\right) \\
& =x^{*}+x_{0}-2 x^{*}=x_{0}-x^{*} .
\end{aligned}
$$

Similarly we show that $x_{0}-v^{*}$ is a solution of equation (1).

Let us assume now that

$$
x_{0}-x^{*}-x^{*}
$$

Then by (29) and (1) we have

$$
x_{0}=2\left(y+B\left(x^{*}, x^{*}\right)\right)=2\left(y+\frac{1}{4} B\left(x_{0}, x_{0}\right)\right) .
$$

which implies

$$
x_{0}=4 y \text {. }
$$

That is

$$
x^{*}=2 y \text {. }
$$

But then by (30) and (1) $2 y=y+B(2 y, 2 y)$ or $4\|B\|\|y\| \geq 1$ since $y \neq 0$ contradicting (ii). This shows (27). Similarly we show (28) and that completes the proof of the theorem. 
We can show the following.

Proposition. Let $B$ be a bounded symmetric bilinear operator on $E \times E$ and suppose that $x_{0}, y \in E$ with $x_{0} \neq 0, x_{0} \neq y$. Assume:

(i) The hypotheses of Theorem 1 are satisfied.

(ii) The inequality (19) is true.

(iii) The inequality

$$
\left\|x_{0}-y\right\|>\frac{1-2 b\left(e-r_{0}\right)-\sqrt{1-4 e b}}{2 b}=R
$$

is true.

Then the solutions $x^{*}$ and $v^{*}$ obtained via Theorems 1 and 2 are distinct.

Proof. Assume that $x^{*}=v^{*}$. The solution $x^{*}$ is such that

$$
\left\|B\left(x^{*}, x^{*}\right)\right\|=\left\|x^{*}-y\right\|
$$

and since $R>r_{0},(32)$ gives

$$
\frac{1-\sqrt{1-4 e b}}{2 b} \geq\left\|x^{*}\right\| \geq \sqrt{\frac{\left\|x_{0}-y\right\|-r_{0}}{b}} .
$$

But then from (33) we deduce

$$
\left\|x_{0}-y\right\| \leq R
$$

contradicting (31).

That completes the proof of the proposition.

Note that under the hypotheses of Theorem 3 and the above proposition it follows immediately that

$$
x_{0}-x^{*} \neq x_{0}-v^{*}
$$

Remarks. (a) It can easily be seen that (6) and (7) can be replaced by the weaker condition

$$
\left\|B\left(x_{0}\right)^{-1} B\right\| \leq q .
$$


(b) If we know the constants $a, b, c, d$ then we may compute the sequence $\left\{t_{n}\right\}, n=0,1,2, \cdots$ before obtaining the sequence $\left\{x_{n}\right\}, n=0,1,2, \cdots$ via the iterative algorithm (2). Therefore the estimates on the distances $\left\|x_{n}-x^{*}\right\|$ and $\left\|x_{n+1}-x_{n}\right\|$ obtained in Theorem 1 may be called apriori error estimates. Moreover the convergence of iteration (2) to a solution $x^{*}$ of equation (1) is only linear. Let us assume that the linear operator

$$
\Gamma_{0}=\left(I-2 B\left(x_{0}\right)\right)^{-1}
$$

exists for some $x_{0} \in E$ and

$$
\left\|\Gamma_{0}\right\| \leq b_{0}, \| \Gamma_{0}\left(x_{0}-y-B\left(x_{0}, x_{0}\right)\left\|\leq \eta_{0}, h_{0}=2 b_{0}\right\| B \| \eta_{0} \leq \frac{1}{2} .\right.
$$

Then the Newton-Kantorovich iteration [7]

$$
z_{n+1}=z_{n}-\left(I-2 B\left(z_{n}\right)\right)^{-1}\left(z_{n}-y-B\left(z_{n}, z_{n}\right)\right), \quad n=0,1,2, \cdots, z_{0}=x_{0}
$$

for solving (1) converges to a unique solution $z_{N}^{*}$ of equation (1) in $U\left(x_{0}, r_{N}\right)$ with

$$
r_{N}=\frac{1-\sqrt{1-4 b b_{0} \eta_{0}}}{2 b b_{0}} .
$$

Moreover the order of convergence is quadratic. However we do not know if $\left\|z_{n}\right\|>p$ for a certain $p \in\left(p_{1}, p_{2}\right)$ whenever $\left\|z_{0}\right\|>p$. That is, we do not know if $\left\|z_{N}^{*}\right\| \not p$ or if $z_{N}^{*} \neq v^{*}$.

It will be shown later that whenever the hypotheses of Theorem 1 are satisfied then the Newton-Kantorovich hypotheses (36) are satisfied also and $x^{*}=z_{N}^{*}$.

That is, if we choose $x_{0}=z_{0}$ with $\left\|x_{0}\right\| \geq p$, then

$$
\left\|z_{N}^{*}\right\| \not p \quad \text { and } \quad z_{N}^{*} \neq v^{*}
$$

even if $z_{n} \geq p$ for some $n, n=0,1,2, \cdots$. Therefore in practice we will prefer to use iteration (37) instead of (2) to find bounded away from zero solution $x^{*}$ of equation (1), since (37) converges faster than (2). However our main concern, that is, the property (38) could only be proved through iteration (2) as the following theorem indicates. 
Theorem 4. Under the hypotheses of Theorem 2 the Newton-Kantorovich iteration (37) for $z_{0}=x_{0}$ converges to a unique solution $z_{N}^{*}$ of equation (1) in $U\left(x_{0}, R_{N}\right)$ and $z_{N}^{*}=x^{*}$. Moreover, if $\left\|z_{0}\right\|>p$ for a certain $p \in\left(p_{1}, p_{2}\right)$ then

$$
\left\|z_{N}^{*}\right\| \geq p
$$

and

$$
\left\|z_{n}-z_{N}^{*}\right\| \leq \frac{1}{2^{n}}\left(2 h_{0}\right)^{2^{n}-1} \eta_{0}, \quad n=0,1,2, \cdots .
$$

Furthermore, the solution $z_{N}^{*}$ can be written as $z_{N}^{*}=x_{0}+h$ where $h$ is a solution of the quadratic equation

$$
h=y_{1}+B_{1}(h, h)
$$

with

$$
y_{1}=\left(I-2 B\left(x_{0}\right)\right)^{-1}\left(B\left(x_{0}, x_{0}\right)+Y-x_{0}\right) \text { and } B_{1}=\left(I-2 B\left(x_{0}\right)\right)^{-1} B \text {. }
$$

Proof. By the Banach lemma, the linear operator

$$
B\left(x_{0}\right)^{-1}-2 I=\left(B\left(x_{0}\right)^{-1}-I\right)-I
$$

is invertible since $\|I\| \cdot\left\|I-B\left(x_{0}\right)^{-1}\right\| \leq c<1$ and

$$
\left\|\left(B\left(x_{0}\right)^{-1}-2 I\right)^{-1}\right\| \leq \frac{1}{1-c} .
$$

The equation (40) has a solution $h$ if

$$
4\left\|y_{1}\right\|\left\|B_{1}\right\| \leq 4\left[\frac{1}{1-c} \cdot d\right]\left[\frac{1}{1-c} \cdot a l\right]<1
$$

which is true by (5). It can easily be scen now that $w^{*}=x_{0}+h$ is a solution of equation (1) if and only if $h$ is a solution of equation (41). The linear operator $\left(I-2 B\left(x_{0}\right)\right)^{-1}$ exists since

$$
\left(I-2 B\left(x_{0}\right)\right)^{-1}=\left(B\left(x_{0}\right)^{-1}-2 I\right)^{-1} B\left(x_{0}\right)^{-1} .
$$


The Newton-Kantorovich hypotheses (36) are now satisfied and by the definition of $r_{N},(19)$ and (20) we deduce that $z_{N}^{*}=w^{*}$. By the uniqueness of the solutions $x^{*}$ and $z_{N}^{*}$ in the balls $U\left(x_{0}, r_{0}\right)$ and $U\left(x_{0}, r_{N}\right)$ it follows that $z_{N}^{*}=x^{*}$ (the balls have the same center).

The rest of the theorem follows from part (ii) of Theorem 2 and Theorem 11.3 in [[7] pp. 142].

All the results obtained in Theorem 2-3 and in the proposition can apply to iteration (37). Note that the result (40) is not known not even for quadratic systmes in $\mathbb{R}^{n}, n>1$.

To cover the cases when $B$ is not symmetric we can state the following theorem whose proof as identical to that of Theorem 1 is omitted.

Theorem 5. Let $B$ be a bounded bilinear operator on $E \times E$ suppose that $x_{0}, y \in E$ with $x_{0} \neq 0, x_{0} \neq y$. Further, let

$\bar{a} \geq\left\|B\left(x_{0}\right)^{-1} B\right\|, \quad \bar{b} \geq\left\|B\left(x_{0}\right)^{-1}(2 \bar{B}-B)\right\|, \bar{c} \geq\left\|B\left(x_{0}\right)^{-1}(2 \bar{B}-B)\left(x_{0}\right)-I\right\|$, and let $A, B$ be defined as

$$
\begin{gathered}
A=\frac{-\left[\bar{b}(\bar{a}+\bar{b}) \bar{t}_{0}+2(\bar{a} \cdot \bar{c}+\bar{b})+\left\{\left[\bar{b}(\bar{a}+\bar{b}) \bar{t}_{0}+2(\overline{a c}+\bar{b})\right]^{2}+4(\overline{a c}+\bar{b}) \bar{t}_{0}\left(\bar{a}^{2}-\bar{b}^{2}\right)\right\}^{\frac{1}{2}}\right.}{2\left(\bar{a}^{2}-\bar{b}^{2}\right)} \\
\bar{t}_{0}=\frac{1-\bar{c}}{\bar{a}+\bar{b}}
\end{gathered}
$$

and

$$
B=\frac{(1+\bar{c})(1-\bar{c})}{2 \bar{a}(\bar{c}+3)}
$$

Assume:

(i) The inverse of the linear operator $B\left(x_{0}\right): E \rightarrow E$ with $B\left(x_{0}\right)(x)=$ $B\left(x_{0}, x\right)$ for all $x \in E$ exists and is bounded;

(ii) The following estimates are true:

$$
\begin{gathered}
\bar{a} \geq \bar{b}, \\
0 \leq c<1,
\end{gathered}
$$




$$
0 \leq d<\bar{t}_{0}-A \text { if } \bar{a}>\bar{b}
$$

and

$$
0 \leq d<\bar{t}_{0}-B \text { if } \bar{a}=\bar{b}
$$

Then

(a) the real sequence $\left\{\bar{t}_{n}\right\}, n=0,1,2, \cdots$ given by

$$
\begin{gathered}
\bar{t}_{n+2}=\bar{t}_{n+1}-\frac{\bar{c}+\bar{b} \bar{t}_{0}-\bar{b} \bar{t}_{n+1}}{1-\bar{a} \bar{t}_{0}+\bar{a} \bar{t}_{n+1}}\left(\bar{t}_{n}-\bar{t}_{n+1}\right), \quad n=0,1,2, \cdots \\
\bar{t}_{1}=B \text { if } \bar{a}>\bar{b}
\end{gathered}
$$

and

$$
\bar{t}_{1}=B \quad \text { if } \quad \bar{a}=\bar{b}
$$

is positive and decreasingly converges to zero.

(b) The sequence $\left\{x_{n}\right\}, n=0,1,2, \cdots$ generated by (2) is well defined, remains in $U\left(x_{0}, \bar{r}_{0}\right)$ and converges to a unique solution $x^{*} \in \bar{U}\left(x_{0}, \bar{r}_{0}\right)$ of equation (1) with $\bar{r}_{0}=\bar{a}^{-1}$.

Moreover, the following estimates are true for all $n=0,1,2, \cdots$

$$
\left\|x_{n+1}-x_{n}\right\| \leq \bar{t}_{n}-\bar{t}_{n+1} \text { and }\left\|x_{n}-x^{*}\right\| \leq \bar{t}_{n} .
$$

Remarks similar to the ones made after the proposition can now easily follow for Thoerem 5 .

The results obtained in the next three examples can also be obtained through the use of iteration (37). However we will only use iteration (2) for demonstrational purposes.

\section{Applications.}

Example 1. Let $E=R^{2}$ and define a bilinear operator on $E$ by

$$
B(w, v)=\left\{\left(w_{1}, w_{2}\right)\left[\begin{array}{ll}
b_{111} & b_{112} \\
b_{121} & b_{122} \\
\hline b_{211} & b_{212} \\
b_{221} & b_{222}
\end{array}\right]\left[\begin{array}{l}
v_{1} \\
v_{2}
\end{array}\right]\right\}
$$




$$
\begin{aligned}
=(B(w))(v) & =\left[\begin{array}{ll}
b_{111} w_{1}+b_{121} w_{2} & b_{112} w_{1}+b_{122} w_{2} \\
b_{211} w_{1}+b_{221} w_{2} & b_{212} w_{1}+b_{222} w_{2}
\end{array}\right]\left[\begin{array}{l}
v_{1} \\
v_{2}
\end{array}\right] \\
& =\left[\begin{array}{ll}
b_{111} w_{1} v_{1}+b_{121} w_{2} v_{1} & +b_{112} w_{1} v_{2}+b_{122} w_{2} v_{2} \\
b_{211} w_{1} v_{1}+b_{221} w_{2} v_{1} & +b_{212} w_{1} v_{2}+b_{222} w_{2} v_{2}
\end{array}\right]
\end{aligned}
$$

Consider the quadratic equation on $E$ given by

$$
w=y+B(w, w)
$$

or equivalently

$$
\begin{aligned}
& w_{1}=\frac{1}{48}-3 w_{1}^{2}+2 w_{1} w_{2}-w_{2}^{2} \\
& w_{2}=-\frac{1}{48}+w_{1}^{2}-2 w_{1} w_{2}-w_{2}^{2}
\end{aligned}
$$

where

$$
\begin{array}{ll}
b_{111}=-3, & b_{221}=-1 \\
b_{112}=1, & b_{222}=-1 \\
b_{121}=1, & y=\left[\begin{array}{l}
y_{1} \\
y_{2}
\end{array}\right], w=\left[\begin{array}{l}
w_{1} \\
w_{2}
\end{array}\right] \\
b_{122}=-1, & y_{1}=\frac{1}{48} \\
b_{211}=1, & y_{2}=-\frac{1}{48} .
\end{array}
$$

For $x \in E$, let $\|x\|=\max _{(i)}\left|x_{i}\right|, i=1,2$. Using the norm on $L(E, E)$ one can define the norm of $B$ on $E$ [10] by

$$
\|B\|=\sup _{\|x\|=1} \max _{(i)} \sum_{j=1}^{2} \sum_{k=1}^{2}\left|\sum_{k=1}^{x} b_{i j k} x_{k}\right|,
$$

from which it follows at once that $\|B\| \leq \max _{(i)} \sum_{i=1}^{2} \sum_{k=1}^{2}\left|b_{i j k}\right|$. Let $x_{0}=\left[\begin{array}{l}-.5 \\ -.5\end{array}\right]$. With the above values it can easily be seen that $B$ is a bounded, symmetric operator on $E$ and

$$
B\left(x_{0}\right)=I, e=d=\|y\|=\frac{1}{48}, b=6, a=1, c=0, r_{0}=\frac{1}{12}, R=.08690776
$$


and

$$
\left\|x_{0}-y\right\|=.520833333 .
$$

According to Theorem 2 (i), equation (44) has a small solution $v^{*} \in \bar{U}\left(0, p_{1}\right)$ which can be found to be $v^{*}=\left[\begin{array}{l}.0200308 \\ .0200308\end{array}\right]$ using the iteration (23) for $v_{0}$ for $v_{0}=y$. We took $v_{8}=v^{*}$. According to Theorem 1, equation (44) has a solution in $U\left(x_{0}, r_{0}\right)$ which can be found to be $x^{*}=\left[\begin{array}{l}-.5200308 \\ -.5200308\end{array}\right]$ using the iteration (2) for $x_{0}=\left[\begin{array}{l}-.5 \\ -.5\end{array}\right]$. We took $x_{9}=x^{*}$ Since $\left\|x_{0}-y_{0}\right\|>R$, it was known before actually computing $v^{*}$ and $x^{*}$ that $x^{*} \neq v^{*}$. Note however that $x^{*}-x_{0}-v^{*}$.

It can easily be seen that $v_{1}^{*}=\left[\begin{array}{c}-.25 \\ .1318813\end{array}\right]$, is the third solution of (44).

Finally, the fourth solution $x_{1}^{*}$ of equation (44) is given by $x_{1}^{*}=x_{0}-v_{1}^{*}$. We have now found all four solutions of equation (44).

A more interesting example is given by the following.

Example 2. Consider the Riccati differential equation

$$
x^{2}(t)+2 z(t) x(t)+y_{1}(t)-\frac{d x}{d t}=0,0 \leq t<T<1, x(0)=0 .
$$

As $E$ takes $C_{0}^{\prime}[0, T]$, the space of all continuously differentiable function $x=x(t)$, such that $x(0)=0$, and as $\hat{E}$ take the space $C[0, T]$ of all continuous real functions. Let us equip the above spaces with the usual sup-norm. That is

$$
\|x\|=\sup _{0 \leq t \leq T}|x(t)| \text { for } x \in E(\text { or } \hat{E}) .
$$

Equation (45) is a quadratic equation of the form (1) with $B\left(x_{1}, x_{2}\right)=$ $B\left(x_{1}\right)\left(x_{2}\right)$ where $B\left(x_{1}\right)$ is a linear operator for fixed $x_{1}$ given by

$$
\begin{aligned}
B\left(x_{1}\right)(w)(t) & =\left[\left[\frac{d}{d t} 2 z\right]^{-1} x_{1} w\right](t) \\
& =\left[\exp \left[\int_{0}^{t} 2 z(q) d q\right]\right] \int_{0}^{t} \exp \left[-\int_{0}^{s} 2 z(q) d q\right] x_{1}(s) w(s) d s
\end{aligned}
$$

for all $w \in E$ and $0 \leq t \leq T$, and

$$
y=\left[\frac{d}{d t}-2 z\right]^{-1} y_{1} .
$$


The linear operator $\frac{d}{d t}-2 z$ is indeed invertible for all $x \in E$, in fact, the inverse transformation $u=\left[\frac{d}{d t}-2 z\right]^{-1} v$ has the explicit representation

$$
u(t)=\left[\exp \left[\int_{0}^{t} 2 z(q) d q\right]\right] \int_{0}^{t} \exp \left[-\int_{0}^{s} 2 z(q) d q\right] v(s) d s, \quad 0 \leq t \leq T,
$$

where $u \in E$ for $v \in \hat{E}$. It can easily be seen that the bilinear operator $B$ defined above is bounded and symmetric. Using definition 3 we deduce for $T=\frac{1}{2}$,

$$
\|B\|=\frac{1}{2} \sup _{0 \leq t \leq T}\left|\left(1-t^{2}\right) \ln \left(1-t^{2}\right)\right| \leq .375 \text { for } z(5)=-\frac{1}{1-t^{2}} .
$$

Take $y_{1}(t)=-.14 \frac{1+t^{2}}{1-t^{2}}$ then easily, $y(t)=-.14 t$ for all $0 \leq t \leq T$ and $\|y\|=$ .07 .

The condition (i) in Theorem 2 is now satisfied. Moreover if the condition (ii) in Theorem 2 is satisfied for some $x_{0}$, then using iterations (2) and (23) we can obtain the solutions $x^{*}$ and $v^{*}$, respectively, with $x^{*} \neq v^{*}$.

Example 3. There are examples of interesting linear operators satisfying condition (26). Indeed, with the notation of the previous example, let us define a linear operator $B(\cdot)$ by $B(v)=\left[\frac{d}{d t}-2 z\right]^{-1}(v)$. Choose $z$ as before and $v(t)=$ $x_{0}(t)=\frac{1+t^{2}}{1-t^{2}}$. It can then easily be seen that $B\left(x_{0}\right)(t)=I(t)=t$ for all $0 \leq t \leq$ $T$, that is $B\left(x_{0}\right)=I$. Therefore the differential equation $\frac{d u}{d t}-2 z(t) u(t)=v(t)$, $u(0)=0$, has the unique solution $u$ given by $u(t)=t, 0 \leq t \leq T$.

Example 4. Consider the scalar equation $x=\delta+\beta x^{2}$ with $\delta, b>0$ and $1-4 \delta \beta>0$.

Let us choose $\frac{1}{2 \beta}<x_{0}<\frac{1+\sqrt{1-4 \delta \beta}}{2 \beta}$. The condition 4,5 and (21) become, respectively,

$$
\begin{gathered}
x_{0} \geq \frac{1}{2 \beta}, \\
\frac{2 \beta+\sqrt{2(1-4 \delta \beta)}}{2 \beta}<x_{0}
\end{gathered}
$$




$$
\begin{gathered}
x_{0}>p \text { for } \quad p \in\left(p_{1}, p_{2}\right), \\
p_{1}=\frac{1-\sqrt{1-4 \delta \beta}}{2 \beta}, \quad p_{2}=\frac{1+\sqrt{1-4 \delta \beta}}{2 \beta} .
\end{gathered}
$$

That is, $x_{0}$ must be chosen such that

$$
\frac{2 \beta+\sqrt{2(1-4 \delta \beta)}}{4 \beta}<x_{0}<\frac{1+\sqrt{1-4 \delta \beta}}{2 \beta}
$$

The large solution of the scalar quadratic equation can now be obtained using iteration (2) for the above choice of $x_{0}$.

\section{References}

[1] I. K. Argyros, "Quadratic equations and applications to Chandrasekhar's and related equations", Bull. Austral. Math. Soc., 32 (1985), 275-292.

[2] ___ "On the approximation of some nonlinear equations", Aeq. Math., 32 (1987), 87-95.

[3] — "An iterative solution of the polynomial equation in Banach space", Bull. Inst. Acad. Sin. 15, 4, (1987), 403-410.

[4] S. Chandrasekhar, "Radiative transfer", Dover Publ., New York, 1960.

[5] H. T. Davis, "Introduction to nonlinear differential and integral equations", Dover Publ., New York, 1962.

[6] L. V. Kantorovich and G. P. Akilov, "Functional analysis in normed spaces", Pergamon Press Publ., 1964.

[7] M. A. Krasnoleskii, et al. "Approximate solution of operator equations", Groningen Publ., 1972.

[8] J. E. McFarland, "An interative solution of the quadratic equation in Banach space." Trans. Amer. Math. Soc. (1985), 824-830.

[9] P. Prenter, "On polynomial operators and equations", Article in Nonlinear Functional Analysis and Applications, pp. 361-398, edited by L. Rall,, Academic Press, New York, 1971.

[10] L. B. Rall, "Quadratic equations in Banach spaces", Rend. Circ. Mat. Palermo 10 (1961), 314-332.

Cameron University, Department of Mathematics, Lawton, OK 73505-6377, U. S. A. 